\title{
REGULARITY OF SEMIGROUP RINGS
}

\author{
JULIAN WEISSGLASS ${ }^{1}$
}

\begin{abstract}
The conditions under which a semigroup ring is regular (in the sense of von Neumann) are investigated. Sufficient conditions are obtained in order that the semigroup ring of an inverse semigroup be regular. Consequences of the regularity of the semigroup ring for the subgroups of the semigroup are established. The two results are then used to find necessary and sufficient conditions for the regularity of the semigroup ring when the semigroup is inverse and a union of groups.
\end{abstract}

1. Introduction. Regularity of group rings has been investigated by Auslander [1], Connell [3] and McLaughlin [4]. In this paper we attempt to extend their results to semigroup rings.

Throughout this paper $R$ will denote an associative ring having an identity element. If $R$ is a ring and $D$ is a semigroup $R D$ will denote the semigroup ring of $D$ over $R$ and $(R D)_{0}$ the contracted semigroup ring if $D$ has a zero. The definition of (contracted) semigroup rings is given in $[8, \S 3]$. It is analogous to the definition of semigroup algebra given in $[2$, p. 159]. Other concepts which are not defined here can also be found in [2]. A ring $S$ is regular (in the sense of von Neumann) if for every $s \in S$ there exists $x \in S$ such that $s x s=s$. An integer $n$ is said to be a unit in a ring $R$ with identity element 1 if $n \cdot 1$ is invertible in $R$.

The result on regularity of group rings (cf. [3, Theorem 3]) is as follows:

TheOREM A. If $G$ is a group and $R$ a ring with identity then $R G$ is regular if and only if $R$ is regular, $G$ is locally finite and the order of every finite subgroup of $G$ is a unit in $R$.

In $\$ 3$ we prove three theorems.

TheOREM 1. Let $D$ be an inverse semigroup, i.e., a regular semigroup in which idempotents commute. $(R D)_{0}$ is regular if

(i) $R$ is regular, 1969.

Presented to the Society, January 23, 1970; received by the editors September 9,

AMS Subject Classifications. Primary 1656, 2090; Secondary 2080, 2092.

Key Words and Phrases. Regular ring, semigroup ring, contracted semigroup ring, group ring, inverse semigroup, Rees matrix semigroup, completely 0 -simple semigroup.

1 The author wishes to thank the referee for his comments. 
(ii) every finite subset of $D$ is contained in a finite inverse semigroup, and

(iii) the order of every finite subgroup of $D$ is a unit in $R$.

A partial converse is obtained for semigroup rings of arbitrary semigroups.

TheOREM 2. Let $D$ be any semigroup. If $(R D)_{0}$ is regular then

(i) $R$ is regular,

(ii) every subgroup of $D$ is locally finite, and

(iii) the order of every finite subgroup of $D$ is a unit in $R$.

Applying Theorems 1 and 2 to a special case we obtain:

THEOREM 3. Let $D$ be an inverse semigroup which is a union of groups $G_{\alpha}, \alpha \in \Omega$. Then $(R D)_{0}$ is regular if and only if

(i) $R$ is regular,

(ii) for every $\alpha \in \Omega, G_{\alpha}$ is locally finite, and

(iii) for every $\alpha \in \Omega$, the order of every finite subgroup of $G_{\alpha}$ is a unit in $R$.

As a consequence of Lemma 2, each of the above theorems remain valid if we replace contracted semigroup ring $(R D)_{0}$ by semigroup ring $R D$.

2. Preliminaries. In this section we isolate the lemmas that are needed to prove the main theorems. The reader may find it expedient to proceed directly to $\S 3$ and refer to the lemmas as needed.

Lemma 1. Let $I$ be a two-sided ideal of a ring $R$. Then $R$ is regular if and only if $R / I$ and $I$ are regular.

Proof. Assume $R$ is regular. Let $r+I \in R / I$. There exists $x \in R$ such that $r x r=r$. Hence $(r+I)(x+I)(r+I)=r x r+I=r+I$. Therefore $R / I$ is regular. Let $a \in I$. There exists $x \in R$ such that $a x a=a$. Now $a(x a x) a=a$ and $x a x \in I$. Hence $I$ is regular. Conversely, assume $R / I$ and $I$ are regular. Let $a \in R$. Since $R / I$ is regular there exists $x+I$ such that $(a+I)(x+I)(a+I)=a+I$. Hence $a x a+I=a+I$, which implies $a x a-a \in I$. Since $I$ is regular, there exists $y \in I$ such that $(a x a-a) y(a x a-a)=a x a-a$. Therefore $a=a(x-x a y a x+y a x+x a y-y) a$. Hence $R$ is regular.

LeMma 2. Let $D$ be a semigroup with a zero element $z$ and suppose that $D \neq\{z\}$.

(i) If $(R D)_{0}$ is regular then both $R$ and $D$ are regular.

(ii) $R D$ is regular if and only if $(R D)_{0}$ is regular. 
Proof. (i) Choose $d \in D, d \neq z$ and choose $r \in R$. The element $r d$ of $(R D)_{0}$ is regular so there exists $x=x_{1} d_{1}+\cdots+x_{n} d_{n} \in(R D)_{0}$ such that $(r d) x(r d)=r d$. Thus $r d=r x_{1} r d d_{1} d+\cdots+r x_{n} r d d_{n} d$. This implies that for some $i, d d_{i} d=d$. Hence $D$ is regular, since for the zero element we have $z z z=z$. Continuing, we have $r=r\left(\sum^{\prime} x_{i}\right) r$ where $\sum^{\prime}$ is over all $i$ such that $d d_{i} d=d$. Therefore $R$ is a regular ring.

(ii) $(R D)_{0}=R D / I$ where $I=\{x \in R D \mid x(d)=0$ if $d \neq z\}$. If $R D$ is regular then by Lemma $1,(R D)_{0}$ is also. Conversely, if $(R D)_{0}$ is regular, by (i) $R$ is regular. Since $I \cong R$, applying Lemma 1 , we see that $R D$ is regular.

In light of Lemma 2, and since a zero can be adjoined to any semigroup, we limit our discussion to the regularity of $(R D)_{0}$ where $D$ is a semigroup with zero.

If $B$ is an ideal of the semigroup $D, D / B$ denotes the Rees factor semigroup of $D$ modulo $B$.

Lemma 3. If $B$ is an ideal of $D$, then $(R D)_{0} /(R B)_{0} \cong(R D / B)_{0}$.

Proof. Let $r_{1} d_{1}+\cdots+r_{n} d_{n}+(R B)_{0}$ be an element of $(R D)_{0} /(R B)_{0}$. Define $\phi\left(r_{1} d_{1}+\cdots+r_{n} d_{n}+(R B)_{0}\right)=r_{1} d_{1}+\cdots+r_{n} d_{n}$ $\in(R D / B)_{0}$. It is easy to verify that $\phi$ is well defined and is a ring isomorphism of $(R D)_{0} /(R B)_{0}$ onto $(R D / B)_{0}$.

Lemma 4. Let $D=D_{1} \supset D_{2} \supset \cdots \supset D_{m} \supset D_{m+1}=\{0\}$ be a principal series for $D$, where 0 is the zero element of $D$. Then $(R D)_{0}$ is regular if and only if $\left(R D_{i} / D_{i+1}\right)_{0}$ is regular for $i=1, \cdots, m$.

Proof. $(R D)_{0}=\left(R D_{1}\right)_{0} \supset\left(R D_{2}\right)_{0} \supset \cdots \supset\left(R D_{m}\right)_{0} \supset\{0\}$ is a finite chain of ideals of $(R D)_{0}$. The lemma is established using induction, Lemma 1 and Lemma 3.

The above lemmas are used in studying the question of the regularity of $(R D)_{0}$ when $D$ is finite. The next and last lemma provides the connection with the known results on group rings.

Lemma 5. Let $D$ be a semigroup and $G$ a subgroup of $D$. If $(R D)_{0}$ is regular then $R G$ is regular.

Proof. Denote by $e$ the identity of $G$. Let $r \in R G$. Since $R G$ is contained in $(R D)_{0}$ in a natural way, there exists $x \in(R D)_{0}$ such that $r x r=r$. Clearly $r($ exe $) r=r$. Write exe $=\sum x(d) d$ with $x(d) \neq 0$. Then $\sum x(d) d=e x e=e($ exe $) e=\sum x(d) e d e$. Hence if $x(d) \neq 0$ we have $d=e d^{\prime} e$, for some $d^{\prime} \in D$. Thus $e d e=e\left(e d^{\prime} e\right) e=e d^{\prime} e=d$. Now write

and let

$$
\text { exe }=\sum x(d) d=\sum_{d \in G} x(d) d+\sum_{d \notin G} x(d) d
$$




$$
y=\sum_{d \in G} x(d) d \quad \text { and } z=\sum_{d \notin G} x(d) d .
$$

Then $e x e=y+z$ and $r=r(e x e) r=r y r+r z r$. We claim that $r z r$ has support disjoint from $G$. Otherwise there exists $d$ in the support of $z$ such that $g_{1} d g_{2}=g_{3}$ for some $g_{1}, g_{2}, g_{3} \in G$. But if $d$ is in the support of $z$, certainly $x(d) \neq 0$ and so $d=e d e=g_{1}^{-1} g_{1} d g_{2} g_{2}^{-1}=g_{1}^{-1} g_{3} g_{2}^{-1} \in G$, which is a contradiction. Since the support of $r y r$ is contained in $G$ and $r$ was chosen in $R G$, we have $r=r y r$.

The following corollary follows immediately from the lemma and Theorem A.

CoRollary. If $(R D)_{0}$ is regular and $G$ is any subgroup of $D$, then $G$ is locally finite and the order of every finite subgroup of $G$ is a unit in $R$.

\section{Proofs.}

Proof of Theorem 1. We sketch the proof since the technique is standard. Condition (ii) allows us to assume, without loss of generality, that $D$ is a finite inverse semigroup and thus has a principal series. Lemma 4 reduces the theorem to proving $(R Q)_{0}$ is regular where $Q$ is a factor of a principal series.

It is well known that if $Q$ is a principal factor of a finite inverse semigroup, then $Q$ is a finite 0 -simple inverse semigroup. $Q$ is 0 -simple by definition and inverse by [6, Corollary 3]. Munn [5, Lemma 4.2] has shown that in this case $Q$ is isomorphic to a $n \times n$ Rees matrix semigroup over a group with zero $G^{0}$ ( $G$ a subgroup of $Q$, and hence of $D$ ) with $n \times n$ identity matrix as sandwich matrix. Using this fact, it is easy to verify (see [8, Lemma 3.1], or [2, Lemma 5.17]) that $(R Q)_{0}$ $\cong(R G)_{n}$ the ring of $n \times n$ matrices over the group ring $R G$. Since $(R)_{n}$ is regular if and only if $R$ is regular [7, Theorem 2.14], it suffices to show that $R G$ is regular. But this follows from Theorem A since $G$ is a finite subgroup of $D$ and hence its order is a unit in $R$.

Proof of Theorem 2. Assume $(R D)_{0}$ is regular. The proof of Lemma 2 shows that $R$ is regular. Lemma 5 and Theorem A establish conditions (ii) and (iii).

It is easy to see that the conditions of Theorem 2 are not sufficient for $(R D)_{0}$ to be regular. If $D$ is the bicyclic semigroup and $R$ is the rationals, $(R D)_{0}$ is not regular and yet $D$ satisfies conditions (ii) and (iii) of Theorem 2 and in fact is inverse.

One can also verify that for $(R D)_{0}$ to be regular it is not necessary that $D$ be inverse. Let $R$ be the rational numbers and $D$ the $2 \times 2$ Rees matrix semigroup over a group with zero $G^{0}$ with sandwich matrix $P$. Choose $G=\left\{1, g \mid g^{2}=1\right\}$ to be the cyclic group of order two and 


$$
P=\left[\begin{array}{ll}
1 & 1 \\
0 & g
\end{array}\right] .
$$

Then $P$ is invertible considered as a matrix over $R G$ so $(R D)_{0}$ is semisimple [2, Theorem 5.20] and hence regular. But $D$ is not inverse.

Proof of Theorem 3. If $(R D)_{0}$ is regular, conditions (i), (ii) and (iii) follows by Theorem 2. Conversely assume (i), (ii) and (iii) hold and that $D$ is an inverse semigroup which is a union of groups. Then, [2, Theorem 4.11], $D$ is a semilattice $\Omega$ of groups $G_{\alpha}(\alpha \in \Omega)$. We will show that conditions (ii) and (iii) of Theorem 1 are satisfied. Let $S=\left\{d_{1}, \cdots, d_{n}\right\}$ be a finite subset of $D$. Let $S_{\alpha}=S \cap G_{\alpha}$. Then only a finite number of the $S_{\alpha}$ are nonempty, say $S_{\alpha_{1}}, \cdots, S_{\alpha_{k}}$. Let $H_{\alpha_{i}}$ be the subgroup in $G_{\alpha_{i}}$ generated by $S_{\alpha_{i}}, i=1, \cdots, k$. Since $G_{\alpha_{i}}$ is locally finite, $H_{\alpha_{i}}$ is finite, $i=1, \cdots, k$. Now the subsemilattice of $\Omega$ generated by $\left\{a_{1}, \cdots, a_{k}\right\}$ is finite, say it is $\left\{\beta_{1}, \cdots, \beta_{l}\right\}$. Hence the set of products of elements from the groups $H_{\alpha_{i}}$ is contained in $\bigcup_{i=1}^{l} G_{\beta_{i}}$. The set of such products being finite, and each $G_{\beta_{i}}$ being locally finite imply that the semigroup generated by $\bigcup_{i=1}^{k} H_{\alpha_{i}}$ is contained in a subsemigroup $D^{\prime}$ which is a finite semilattice of finite groups. Now the idempotents of $D^{\prime}$ commute, since they are a subset of the idempotents of $D$. Hence $D^{\prime}$ is a finite inverse subsemigroup of $D$ containing $\left\{d_{1}, \cdots, d_{n}\right\}$. This establishes condition (ii) of Theorem 1. Condition (iii) of Theorem 1 holds since it is easily verified that any subgroup of $D$ is a subgroup of some $G_{\alpha}$. Hence $(R D)_{0}$ is regular.

\section{BibliogRAPHY}

1. M. Auslander, On regular group rings, Proc. Amer. Math. Soc. 8 (1957), 658664. MR 19, 390.

2. A. H. Clifford and G. B. Preston, The algebraic theory of semigroups. Vol. 1, Math. Surveys, no. 7, Amer. Math Soc., Providence, R. I., 1961. MR 24 \#A2627.

3. I. G. Connell, On the group ring, Canad. J. Math. 15 (1963), 650-685. MR 27 \#3666.

4. J. E. McLaughlin, A note on regular group rings, Michigan Math. J. 5 (1958), 127-128. MR 21 \#1986.

5. W. D. Munn, Matrix representations of semigroups, Proc. Cambridge Philos. Soc. 53 (1957), 5-12. MR 18, 489.

6. W. D. Munn and R. Penrose, A note on inverse semigroups, Proc. Cambridge Philos. Soc. 51 (1955), 396-399. MR 17, 10.

7. J. von Neumann, Continuous geometry, Princeton Math. Series, no. 25, Princeton Univ. Press, Princeton, N. J., 1960. MR $22 \# 10931$.

8. J. Weissglass, Radicals of semigroup rings, Glasgow Math. J. 10 (1969), 85-93.

University of California, Santa Barbara, California 93106 